\title{
REVIEW
}

\section{Oligosaccharides in Milk: Their Benefits and Future Utilization}

\author{
T. Urashima ${ }^{a} \&$ E. Taufik ${ }^{a} b$ \\ aGraduate School of Animal Husbandry, Animal and Food Hygiene Program, \\ Obihiro University of Agriculture and Veterinary Medicine \\ Obihiro Shi Inada Cho Nishi 2-11, Hokkaido, 080-8555, Japan \\ bDepartment of Animal Production Science and Technology, Faculty of Animal Science, \\ Bogor Agricultural University \\ Jln. Agatis Kampus IPB Darmaga Bogor, 16680, Indonesia \\ (Received 16-11-2010; accepted 28-12-2010)
}

\begin{abstract}
The percentage of carbohydrate in the milk/colostrum of the mammalian is range from trace to over $10 \%$, of which disaccharide lactose $(\mathrm{Gal}(\beta 1-4) \mathrm{Glc})$ is usually constitutes the major part. Apart from the lactose (Gal( $\beta 1-4) G l c ;$ Gal, D-galactose; Glc, D-glucose), the rest of carbohydrate components is composed of variety of sugars, commonly named as milk oligosaccharides. Human mature milk and colostrum contain $12 \sim 13 \mathrm{~g} / 1$ and $22 \sim 24 \mathrm{~g} / 1$ of oligosaccharides, respectively. In contrast, bovine colostrum contains more than $1 \mathrm{~g} / \mathrm{l}$ oligosaccharides and this concentration rapidly decreases after $48 \mathrm{hr}$ post partum. Most of human milk oligosaccharides (HMO) are resistant to digestion and absorption within the small intestine. Therefore they can reach the infant colon, where they can act as prebiotics that stimulate the growth of beneficial microorganisms such as various species of Bifidobacterium. They can also act as receptor analogues that inhibit the attachment of pathogenic microorganisms to the infant's colonic mucosa. A small part of the milk oligosaccharides is absorbed intact into the circulation and it has been hypothesized that these may act as immunomodulators. Generally, the bovine milk oligosaccharides are believed not to be absorbed by human adults or infants, thus making them available to be utilized as prebiotics or anti-infection materials. The colostrum of cows and other domestic farm animals is a potential source of free oligosaccharides, and oligosaccharides isolated from these natural sources can be utilized as functional foods or animal feedstuffs on the industrial scale.
\end{abstract}

Key words: milk, oligosaccharides, prebiotic, Bifidobacterium

\section{INTRODUCTION}

Human milk oligosaccharides generally represent a large proportion of the total solids with components. Human colostrum and mature milk contain 22-24 g/ and 12-13 g/ of oligosaccharides, respectively; this oligosaccharide fraction is the third largest solid components, after lactose and lipid (Newburg \& Neubauer, 1995). The oligosaccharide content of human milk is about 20-fold higher than that of bovine milk and is even higher than that of bovine colostrum (Veh et al., 1981; Nakamura et al., 1998). Human milk is also considered to be unique in its high content of complex fucosylated and sialylated

\footnotetext{
* Corresponding author: e-mail: urashima@obihiro.ac.jp
}

oligosaccharides (Kunz et al., 1999). In this review, we will introduce the structural feature of human milk oligosaccharides, on their fate within the gastrointestinal tract as well as on their possible biological functions as prebiotics, anti infection agents and immunomodulation factors. In addition, we will discuss the possibility of the commercial utilization of bovine milk oligosaccharides and those of other domestic farm animals.

\section{THE CHEMICAL STRUCTURES AND THEIR QUANTITATIVE ASPECTS OF HUMAN MILK OLIGOSACCHARIDES}

The structures of at least 115 human milk oligosaccharides (HMOs) have been determined to date (Urashima et al., in press), while as many as 200 different oligosaccharides have been separated and studied by microfluidic high performance liquid chromatography 
(HPLC) - chip mass spectrometry (MS) (Ninonuevo et al., 2006). The 115 human milk oligosaccharides, the structures of which have been determined to date, can be grouped into 13 series based on their core units as in Table 1 (Urashima et al., in press). The many variations of the oligosaccharides are constructed by the addition of a Neu5Ac $\alpha 2-3 / 2-6$ residue to Gal or GlcNAc, and of Fuc $\alpha 1-2 / 1-3 / 1-4$ to Gal, GlcNAc or a reducing Glc of the core units.

The main structural features of human milk oligosaccharides are the presence of oligosaccharides containing the type I unit ( $\mathrm{Gal}(\beta 1-3) \mathrm{GlcNAc})$, as well as those containing the type II unit $(\mathrm{Gal}(\beta 1-4) \mathrm{GlcNAc})$. The milk oligosaccharides of other species mostly have the type II but not the type I unit (Urashima et al., 2001; Urashima et al., 2007). The many varieties of oligosaccharides in human milk and colostrum are produced by the addition of Neu5Ac and/or Fuc residues to these two units.

The great variety of oligosaccharides in human milk depends on the activity of many different specific enzymes in the lactating gland (Urashima et al., in press). For example, a $\alpha 1-2$-fucosyltransferase is expressed in about $77 \%$ of all Caucasian women; these are classified as secretors. Therefore, the oligosaccharides in the milk from these women are characterized by the presence of $\alpha 1$-2-fucosylated components, e.g.,

Table 1 . The 13 core structures of human milk oligosaccharides

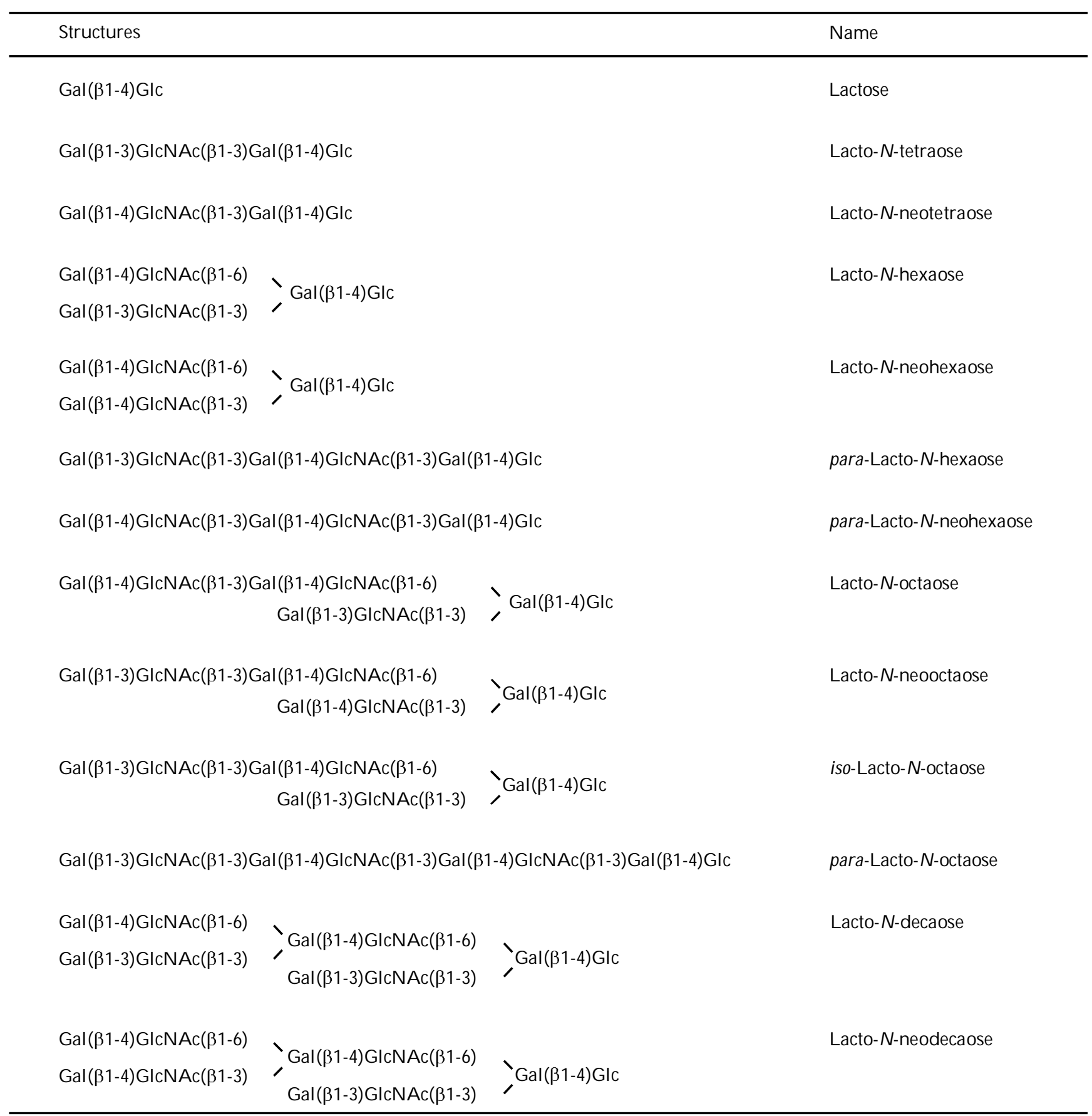


Fuc $(\alpha 1-2)$ Gal( $\beta 1-4)$ Glc (2'-fucosyllactose: 2'-FL), Fuc $(\alpha 1-$ 2)Gal( $\beta 1-3)$ GlcNAc( $\beta 1-3)$ Gal $(\beta 1-4)$ Glc(lacto- $N$-fucopentaose I : LNFP I), or more complex oligosaccharides, all possessing Fuc $(\alpha 1-2) \mathrm{Gal}(\beta 1-3) \mathrm{GlcNAc}$ residues. In the mammary glands of Lewis $(a+b-)$ individuals, who produce nonsecretor milk, this enzyme activity is absent; as a result, the major fucosylated oligosaccharide is $\operatorname{Gal}(\beta 1-3)[\mathrm{Fuc}(\alpha 1-4)] \mathrm{GlcNAc}(\beta 1-3) \mathrm{Gal}(\beta 1-4) \mathrm{Glc}$ (lacto- $N$-fucopentaose II : LNFP II). This pattern of HMO is found in about $20 \%$ of the population. In about $5 \%$ of the population who belong to blood group Lewis (a-b-), LNFP II is absent from the milk because of the absence of $\alpha 1$-4-fucosyltransferase activity (Urashima et al., in press).

The studies on the quantification of each of the oligosaccharides, in terms of their concentration in human colostrum and mature milk, have only recently been undertaken. Milk oligosaccharides can be quantified using reverse - phase or normal - phase HPLC subsequent to pre- or post - column labeling techniques. Derivatizations are often performed by condensation with 2-aminopyridine (PA) (Asakuma et al., 2008), 1-methyl-3-phenyl-5-pyrazolone (PMP) (Asakuma et al., 2007, 2008), anthranilic acid (Leo et al., 2009, 2010), or benzoic anhydride (Chaturvedi et al., 2001) to the reducing end of the sugar aldehyde. They can also be quantified by high $\mathrm{pH}$ anion exchange chromatography (HPEAC-PAD) without derivatization (Thurl et al., 1996; Coppa et al., 1999; Kunz et al., 1996). Sialyl oligosaccharides can be quantified by capillary electrophoresis using phosphate buffer containing sodium dodesyl sulfoxide (SDS) (Shen et al., 2000; Bao et al., 2007).

When the concentrations of representative neutral HMO including 2'-FL, Gal( $\beta 1-4)[\mathrm{Fuc}(\alpha 1-3)] \mathrm{Glc}$ (3-FL), LDFT, LNT, LN $n$ T, LNFP-I, LNFP-II, LNFPIII, LNDFH-I or LNDFH-II and of representative acidic HMO including Neu5Ac( $\alpha 2-6) \mathrm{Gal}(\beta 1-4) \mathrm{Glc}$ $\left(6^{\prime}-\mathrm{SL}\right)$, Neu5Ac $(\alpha 2-3) \mathrm{Gal}(\beta 1-4) \mathrm{Glc}\left(3^{\prime}-\mathrm{SL}\right)$, Neu5Ac $(\alpha 2-$ 3) Gal $(\beta 1-3)$ GlcNAc $(\beta 1-3)$ Gal $(\beta 1-4)$ Glc (LST-a), Gal $(\beta 1-$ 3) [Neu5Ac $(\alpha 2-6)]$ GlcNAc( $\beta 1-3)$ Gal $(\beta 1-4)$ Glc(LST-b), Neu5Ac( $\alpha 2-6)$ Gal $(\beta 1-4)$ GlcNAc( $\beta 1-3)$ Gal $(\beta 1-4)$ Glc (LSTc), $\operatorname{Neu} 5 \mathrm{Ac}(\alpha 2-3) \mathrm{Gal}(\beta 1-3)[\mathrm{Neu} 5 \mathrm{Ac}(\alpha 2-6)] \mathrm{GlcNAc}(\beta 1-$ 3) Gal( $\beta 1-4) G l c(D S L N T)$, Fuc $(\alpha 1-2) \operatorname{Gal}(\beta 1-3)[\mathrm{Neu} 5 \mathrm{Ac}(\alpha 2-$ $6)] G l c N A c(\beta 1-3) G a l(\beta 1-4) G l c(s L N F P-I)$ and Neu5Ac $(\alpha 2-3)$ Gal $(\beta 1-3)[F u c(\alpha 1-4)$ ]GlcNAc $(\beta 1-3)$ Gal $(\beta 1-4)$ Glc (sLNFP-II) were quantified in the colostra of Japanese women, the predominant oligosaccharides were shown to be 2'-FL, LNFP-I, LNDFH-I and LNT, in that order (Asakuma et al., 2007, 2008). Assuming that the total oligosaccharide concentration in colostrum was 22 24 g1 (Newburg \& Neubauer, 1995), then these four oligosaccharides can be calculated to constitute $25 \%$ to $33 \%$ of the total. It has been shown that the above four $\mathrm{HMO}$ are also predominant oligosaccharides in human mature milk (Chaturvedi et al., 2001; Thurl et al., 1996). The concentrations of each of the acidic oligosaccharides in colostra or in mature milk were lower than those of the four neutral oligosaccharides; the most predominant acidic oligosaccharide was LST-c, followed by DSLNT, 6'-SL, 3'-SL and LST-a in that order (Thurl et al., 1996).

It is notable that threeffourth of the prominent oligosaccharides in human milk or colostra are of type I, which contain Gal( $\beta 1-3)$ GlcNAc (lacto-N-biose I) at their non-reducing termini (Asakuma et al., 2008). The concentration of LNT, a type I oligosaccharide, was found to be 3 to 4 times higher than that of $\mathrm{LN} n \mathrm{~T}$, which is a type II oligosaccharide containing the Gal( $\beta 1-4)$ GlcNAc ( $N$-acetyllactosamine) group at its non-reducing terminus (Asakuma et al., 2008). Urashima et al. (2001, 2007) have been characterizing the milk oligosaccharides of many mammalian species; they have found that the milk or colostra of most of these species contain only type II oligosaccharides, notable exceptions being chimpanzee, bonobo and orangutan whose milk or colostrum contains both types I and II. However, type II oligosaccharides predominate over type I in the milk or colostra of these apes (Urashima et al., 2009), suggesting that the predominance of type I milk oligosaccharides is a characteristic feature of human milk.

\section{DIGESTION AND ABSORPTION OF MILK OLIGOSACCHARIDES IN THE GASTROINTESTINAL TRACT}

When infants consume milk, the free lactose therein is split into galactose and glucose by intestinal lactase (neutral $\beta$-galactosidase, lactose phlorizin hydrolase), an enzyme that is located in the membrane of the microvilli of the brush border of the small intestine. The two monosaccharides are transported into the enterocytes by a specific mechanism, whereupon the glucose enters the circulation and is used as an energy source while most of the galactose is converted to glucose in the liver, to be used as an energy source as well.

Much less is known about the exact metabolic fate of human milk oligosaccharides. They are resistant to enzymatic hydrolysis by the intestinal lactase of the brush border (Engfer et al., 2000), and there is evidence that the major part of oligosaccharides passes through the small intestine without degradation and enters the colon, where they are fermented by colonic bacteria (BrandMiller et al., 1998; Newburg, 2000). Evidently, the brush border of the small intestine does not contain enzymes, such as sialidase, fucosidase or $\mathrm{N}$-acetylhexosaminidase that can remove sialic acid, fucose, $\mathrm{N}$-acetylglucosamine residues, respectively, from the lactose or other core units of the milk oligosaccharides. A small fraction of human milk oligosaccharides is absorbed intact, perhaps by receptor-mediated endocytosis (Gnoth et al., 2001), some of which are excreted in the urine. It is unclear what proportion and exactly which of the consumed milk oligosaccharides are absorbed, but there is an evidence suggesting that circulating oligosaccharides may have immunological effects on endothelial cells (Rudloff et al., 1996).

\section{BIOLOGICAL SIGNIFICANCES OF HUMAN MILK OLIGOSACCHARIDES}

\section{Bifidobacterium Growth Stimulation}

Bifidobacteria constitute around $95 \%$ to $99.9 \%$ of the total colonic microflora within one week after birth in breast fed infants. This proportion is lower in the flora of 
bottle fed infants even when prebiotic oligosaccharides such as lactulose, galacto oligosaccharides or fructo oligosaccharides are present in the milk replacer (Rotimi \& Duerden, 1981; Benno \& Mitsuoka, 1986). It was suggested that milk components stimulate the growth of colonic bifidobacteria. As mentioned above, human milk oligosaccharides are believed to act as prebiotics that stimulate the growth of bifidobacteria in the lower intestine; this reduces the colonic $\mathrm{pH}$, which would tend to inhibit the growth of pathogenic bacteria such as $E$. coli.

Which of the more than 100 different human milk oligosaccharides do stimulate the growth of the infant's colonic bifidobacteria? Kitaoka et al. (2005) have proposed that milk oligosaccharides which contain lacto-N-biose I (LNB, Gal( $\beta 1-3)$ GlcNAc) are preferential growth factors for some species of Bifidobacterium. This hypothesis is based on the published genome information relating to bifidobacteria and the cloning of glycohydrolases, a glycophosphorylase and an ABC transporter from a strain of $B$. longum. The hypothesis was initially based on the discovery of an enzyme, in cell extracts of B. bifidum, that phosphorolyses lacto-N-biose, the products being galactose-1-phosphate and N-acetylglucosamine (Derensy-Dron et al., 1999): this enzyme was subsequently purified, and its gene, $\ln p A$, from $B$. longum was cloned (Kitaoka et al., 2005). The $\operatorname{lnp} A$ gene was found to be located in a novel operon for galactose metabolism which also includes genes for a mucin desulfatase and for Gal-1-P uridyl transferase and UDP-Glc-4epimerase as well as a gene for an ATP-binding cassette sugar transporter (Kitaoka et al., 2005). In addition it was demonstrated that lacto-N-biose phosphorylase can also utilise galacto-N-biose (GNB, Gal( $\beta 1-3)$ GalNAc), which is a mucin core unit, as a substrate, but not N-acetyllactosamine (LacNAc, Gal $(\beta 1-4)$ GlcNAc) (Kitaoka et al., 2005).

The high content and increase in the concentrations of LNB containing oligosaccharides such as LNT, LNFP I and LNDFH I in early stage milk/colostrum could affect the formation of bifidus flora in the infant colon significantly. The metabolism of LNFP I, which is a dominant human milk oligosaccharide, by B. bifidum JCM1254 was clarified by the cloning of an $\alpha$-fucosidase (Katayama et al., 2004), which catalyses the release of non reducing fucose from LNFP I, and a lacto-N-biosidase (Wada et al., 2008), which catalyses the hydrolysis of LNT to produce LNB and lactose, and by the purification and crystallization of an ABC type transporter (Suzuki et al., 2008) which delivers LNB through the cell membrane. Since lacto-N-biose phosphorylase was purified from an extract of this bifidobacterial strain, it has been suggested that its $\alpha$-fucosidase produces LNT from LNFP I, while its lacto-N-biosidase liberates LNB from LNT, which is followed by the uptake of LNB through the cell membrane, the LNB is then metabolized to galactose-1phosphate and $\mathrm{N}$-acetylglucosamine. A possible metabolic pathway for the $\mathrm{N}$-acetylglucosamine, produced from LNFP I or LNT (see above) is suggested, based on the analysis of gene products of the galactose operon of B. longum ssp. longum NCC2705; N-acetylglucosamine is converted to $\mathrm{N}$-acetylglucosamine-1-phosphate by the novel enzyme $\mathrm{N}$-acetylhexosamine 1-kinase and then to UDP-N-acetylglucosamine by UDP-glucose hexose 1-phosphate uridyltransferase, finally entering the metabolic pathway of amino sugars (Nishimoto \& Kitaoka, 2007).

On the other hand, growth tests with B. longum ssp infantis ATCC 15697, B. longum ssp. longum ATCC 15707, B. adolescentis ATCC 15703, B. breve ATCC 27539 or B. bifidum ATCC 29521 were performed using a medium containing $1 \%$ human milk oligosaccharides but no lactose, which was incubated at $37^{\circ} \mathrm{C}$ for $350 \mathrm{hr}$ (Ward et al., 2007). This resulted in a cell density of B. longum ssp. infantis strains that was three times higher than that of other strains. When oligosaccharide and monosaccharide profiles in the medium containing human milk oligosaccharides were studied using high performance thin layer chromatography, a clear change was observed after incubation with $B$. longum ssp. infantis, when compared with the control, which was the medium with no inoculation. This change was not found in the medium after incubation with $B$. breve and adolenscentis. These results showed that $B$. longum ssp. infantis is able to grow with human milk oligosaccharides as the only carbon source.

The degradation of human milk oligosaccharides was studied during the growth of B. longum ssp. infantis ATCC15697, B. breve ATCC15700 or B. longum ssp. longum DJO010A in broth containing oligosaccharides but no lactose by observation of the molecular mass of ions in FTICR-MS (LoCascio et al., 2007). In this work, tri to heptasaccharides were completely degraded during the growth of B. longum ssp. infantis ATCC 15697 over 25 and $50 \mathrm{hr}$ of incubation, while higher oligosaccharides were partially degraded.

The genome of B. longum ssp. infantis ATCC15697 was fully sequenced to permit investigations into the genetic basis and molecular mechanisms underlying this phenotype. Many of its genomic features encode enzymes that are active on human milk oligosaccharides and include a novel $43 \mathrm{kbp}$ region dedicated to oligosaccharide utilization (Sela et al., 2008). Subsequent biochemical and molecular characterization of human milk oligosaccharide - related glycosidases and transport proteins have further resolved the mechanism by which this strain imports and catabolizes milk oligosaccharides. The glycosidases were identified to be sialidase, fucosidase, $\beta$-galactosidase, $\beta$-hexosaminidase and LNB phosphorylase, but lacto-N-biosidase was not found. This approach suggested the existence of a pathway for the metabolism of milk oligosaccharides in which they are transported into the bifidobacterial cell and then degraded from the non-reducing end by the above glycosidases.

\section{Anti-pathogenic Agents}

It is assumed that HMOs are synthesized by the actions of several glycosyltransferases, which usually catalyze the biosynthesis of carbohydrate moieties of glycoproteins and glycolipids. Therefore it is thought that the structures of the non-reducing ends of $\mathrm{HMO}$ are similar to those of the non-reducing ends of the 
sugar chains of glycoconjugates, which are found on the surface of epithelial cells. It was known that infection by many bacteria and viruses starts by binding to particular sugar chains of glycoconjugates on the surface of cells of the mucous epithelium of the digestive and respiratory tracts. Therefore, human milk oligosaccharides might be useful for elucidating the structures of the sugar chains on the surface of epithelial cells that are the targets of specific bacteria or viruses.

Ruiz-Palacios et al. (2003) found that HMO containing Fuc( $\alpha 1-2) \mathrm{Gal}(\beta 1-4) \mathrm{GlcNAc}$ (H-2 antigen epitope) at their non-reducing termini inhibit the attachment of Campylobacter jejuni to colonic epithelial cells. This organism causes diarrhea and paralysis of motor nerves in infants. A universal correlation has been observed between the breast milk concentration of 2'-FL, which is usually the most prominent oligosaccharide in secretor donor's milk, and the frequency of diarrhea in breast fed infants, supporting the view that 2 '-FL reduces the pathogenicity of $C$. jejuni.

An interaction of Helicobacter pylori with sialylated glycans has been reported. H. pylori is a Gram-negative bacterium, resides in the gastric mucosa and adheres to the epithelial cells lining the stomach. This organism infects around $50 \%$ of the world population, with a higher incidence in developing countries. H. pylori is associated with the development of peptic ulcers, mucosa-associated lymphoid-tissue (MALT) lymphoma and gastric adenocarcinoma. The preferred interaction is with $\alpha 3$-linked sialic acid; glycans having $\alpha 6$-linked Neu5Ac are non-binding. For example, 50\% inhibition by $H$. pylori of hemagglutination of human erythrocytes was observed at a low concentration of some sialylated saccharides. The data showed that S-3 PG (Neu5Ac $(\alpha 2-$ 3) Gal $(\beta 1-4)$ GlcNAc( $\beta 1-3)$ Gal $(\beta 1-4)$ Glc-Cer $)$, Neu5Ac $(\alpha 2-$ 3) Gal $(\beta 1-4)[\mathrm{Fuc}(\alpha 1-3)]$ GlcNAc, $\quad 3$ - $N$-acetylneuraminyl$N$-acetyllactosamine (Neu5Ac $(\alpha 2-3) \mathrm{Gal}(\beta 1-4) \mathrm{GlcNAc})$ as well as $3-N$-acetylneuraminyl-lacto- $N$-neotetraose $(\mathrm{Neu} 5 \mathrm{Ac}(\alpha 2-3) \mathrm{Gal}(\beta 1-4) \mathrm{GlcNAc}(\beta 1-3) \mathrm{Gal}(\beta 1-4) \mathrm{Glc})$ all bound to $H$. pylori CCUG17874 at similar strength. 3'-SL also bound to this organism but its binding ability was somewhat weaker than that of the above saccharides. It has also been reported that LST a, a human milk oligosaccharide, was able to bind to another strain, H. pylori J99 (Johansson et al., 2005).

The binding of 3 '-SL to $H$. pylori CCUG17874 is noteworthy because this saccharide is found in human milk and bovine colostrum. Asakuma et al. (2007) found that at the start of lactation the concentration of $3^{\prime}$-SL in human colostrum was $360 \mathrm{mg} /$, similar to that of $6^{\prime}-\mathrm{SL}$. However, the concentration of 3 '-SL decreased during the subsequent two days of lactation, whereas that of 6 '-SL did not. This suggests that, very early stage in lactation, 3'-SL may be more significant in the prevention of transmission of $H$. pylori from mother to infant than later on.

Recent studies on the ability of various fractions of human milk oligosaccharides to inhibit the adhesion of three intestinal microorganisms (enteropathogenic E. coli serotype 0119, Vibrio cholerae and Salmonella fyris) to differentiated Caco- 2 cells have shown that the acidic fraction had an anti-adhesive effect on all three patho- genic strains. The neutral high molecular weight fraction significantly inhibited the adhesion of E. coli 0119 and $V$. cholerae, but not that of $S$. fyris; the neutral low molecular weight fraction was effective toward E. coli 0119 and $S$. fyris but not $V$. cholerae (Coppa et al., 2006). This demonstrated that human milk oligosaccharides inhibit the adhesion to epithelial cells not only of common pathogens such as E. coli but also of other aggressive bacteria such as $V$. cholerae and S. fyris. Thus, oligosaccharides may be important factors in human milk that defend against acute diarrhea in breast-fed infants.

Furthermore, it has been reported that the human milk oligosaccharides fraction, at a concentration of 0.5 $\mathrm{g} /$, reduced the binding of the HIV-1 envelope glycoprotein gp120 to dendritic cell - specific ICAM3 - grabbing non - integrin (DC-SIGN) in human dendritic cells by more than $60 \%$. In addition, the binding of gp120 to Raji cells, which expressed DC-SIGN, was reduced by more than $60 \%$. It is worth noting that mother to child transmission accounts for more than $40 \%$ of all HIV-1 infections in children, with breast-feeding being the predominant postnatal transmission route, especially in developing countries. However, a majority of breast-fed infants born to HIV-positive mothers remain uninfected despite continuous exposure to the virus over many months. Viral entry across the infant's mucosal barrier is partially mediated by binding of gp120 to DC-SIGN on human dendritic cells (DC). It has been hypothesized that human milk oligosaccharides, when they reach DC in the colon, bind to DC-SIGN and inhibit the transfer of HIV to CD4 + T lymphocytes. It has also been suggested that this inhibition of HIV-gp120 binding to DC-SIGN is caused by oligosaccharides carrying multiple Lewis epitopes (Hong et al., 2009).

\section{Immuno - modulating Effect}

It is thought that the immuno - modulating effect of human milk oligosaccharides in infants is acquired while the oligosaccharides are circulating after their absorption, or by immuno stimulation in the colon produced by growth-stimulated beneficial bacteria. Although unequivocal detection of human milk oligosaccharides in the blood of infants has not yet been reported (Bode, 2006), it nevertheless seems very likely on the basis of their observed urinary excretion (Rudloff et al., 1996; Obermeier et al., 1999) that small amounts of intact milk oligosaccharides are normally absorbed from the gastrointestinal tract, and that they are transported into the systemic circulation.

It follows that they may alter protein-carbohydrate interactions also at a systemic level. For example, recent studies suggest that human milk oligosaccharides interfere with the adhesion of neutrophils to vascular endothelial cells (Klein et al., 2000) and platelets (Bode et al., 2004). These effects appear to be based on the structural resemblance of some human milk oligosaccharides to the glycoprotein ligands of selectins. Selectins are trans-membrane proteins that are involved in cell-cell interactions in the immune system. P-selectin mediates leukocyte deceleration (rolling) on activated endothelial cells and initiates leukocyte extravasation at sites of in- 
flammation. P-selectin is also involved in the formation of platelet-neutrophil complexes (PNC), a sub-population of highly activated neutrophils primed for adhesion, phagocytosis and enhanced production of reactive oxygen species. Recent studies suggest that oligosaccharides containing sialyl $\mathrm{Le}^{\mathrm{x}}$ or its stereoisomer sialyl $\mathrm{Le}^{\mathrm{a}}$, which resemble the P-selectin ligand, inhibit the binding of selectin ligands to the surface of endothelial cells and platelets; this interferes with the formation of PNC, the effect of which is anti-inflammatory. The following oligosaccharide fractions were tested in vitro to establish whether they reduce leukocyte deceleration on U937 cells, which express the P-selectin ligand: total human milk oligosaccharides, neutral oligosaccharides, total acidic oligosaccharides, neutral oligosaccharides with a polymerization degree of 4 , fucosylated oligosaccharides and disialyl lacto- $N$-tetraose. The acidic oligosaccharide fraction produced a slight but definite reduction of P-selectin ligand binding, similar to that of standard sialyl $\mathrm{Le}^{\mathrm{x}}$, whereas the total neutral oligosaccharides and neutral fucosylated oligosaccharides fractions did not (Schumacher et al., 2006). These results support the notion of anti-inflammatory effects of acidic human milk oligosaccharides.

\section{MILK OLIGOSACCHARIDES OF THE COW AND OTHER DOMESTIC FARM ANIMALS AND THEIR UTILIZATION IN THE FUTURE INDUSTRY}

Nakamura et al. (2003) and Gopal \& Gill (2000) stated that bovine colostrum collected immediately after parturition contains more than $1 \mathrm{~g} / \mathrm{L}$ of oligosaccharides, but the mature milk contains only small amounts. Although 25 bovine oligosaccharides structures isolated from the colostrum have been completely characterized (Table 2), as many as 39 oligosaccharides have been detected using a combination of nanoelectrospray Fourier transform ion cyclotron resonance (nESI-FTICR) mass spectrometry and matrix-assisted laser desorption/ ionization Fourier transform ion cyclotron resonance (MALDI-FTICR) mass spectrometry (Tao et al., 2008). Some bovine oligosaccharides include those that have $N$ acetyllactosamine $(\mathrm{Gal}(\beta 1-4) \mathrm{GlcNAc})$ instead of lactose at their reducing ends, such as free $N$-acetyllactosamine or $6^{\prime}-N$-acetylneuraminyl-N-acetyllactosamine $\left(6^{\prime}-\mathrm{SLN}\right.$, $\operatorname{Neu} 5 \mathrm{Ac}(\alpha 2-6) \mathrm{Gal}(\beta 1-4) \mathrm{GlcNAc})$ and others. There is a difference in this respect between bovine and human milk oligosaccharides, insofar as almost all HMO contain a lactose unit at their reducing ends.

Most of the acidic oligosaccharide fraction of bovine colostrum consists of $3^{\prime}-\mathrm{SL}, 6^{\prime}-\mathrm{SL}, 6^{\prime}-\mathrm{SLN}$ and DSL (Neu5Ac $(\alpha 2-8) \operatorname{Neu} 5 \mathrm{Ac}(\alpha 2-3) \mathrm{Gal}(\beta 1-4) \mathrm{Glc})$, with $3^{\prime}-\mathrm{SL}$ constituting $70 \%$ of this total (Tao et al., 2008). There is a difference between human and cow in the ratio of $3^{\prime}-\mathrm{SL}$ to $6^{\prime}-\mathrm{SL}$, in that $6^{\prime}$-SL predominates over $3^{\prime}$-SL in human milk/colostrum. The levels of the acidic oligosaccharides were maximal immediately after parturition, rapidly decreasing by $48 \mathrm{~h}$ post-partum (Nakamura et al., 2003).

Since bovine colostrum contains some oligosaccharides, which are mainly acidic, and mature bovine milk also contains them, although at lower concentrations, it can be expected that oligosaccharides derived from
Table 2. Bovine milk oligosaccharides

\begin{tabular}{|c|c|c|}
\hline No & Oligosaccharide & Structure \\
\hline \multicolumn{3}{|c|}{ Neutral oligosaccharides } \\
\hline \multirow[t]{2}{*}{1} & $\mathrm{~N}$-Acetylgalactosar & flglucose \\
\hline & & GalNAc $(\beta 1-4)$ Glc \\
\hline 2 & LacNAc & Gal(ß1-4)GlcNAc \\
\hline 3 & Le $^{x}$ trisaccharide & $\begin{array}{r}\operatorname{Gal}(\beta 1-4) \mathrm{GlcNAc} \\
\operatorname{Fuc}(\alpha 1-3)\end{array}$ \\
\hline 4 & $\alpha 3^{\prime}-$ GalNAcL & $\operatorname{GalNAc}(\alpha 1-3) \operatorname{Gal}(\beta 1-4) \mathrm{Glc}$ \\
\hline 5 & Isoglobotriose & $\operatorname{Gal}(\alpha 1-3) \mathrm{Gal}(\beta 1-4) \mathrm{Glc}$ \\
\hline 6 & $\beta 3^{\prime}-G L$ & $\operatorname{Gal}(\beta 1-3) \mathrm{Gal}(\beta 1-4) \mathrm{Glc}$ \\
\hline 7 & $\beta 4 '-G L$ & $\operatorname{Gal}(\beta 1-4) \mathrm{Gal}(\beta 1-4) \mathrm{Glc}$ \\
\hline 8 & $\beta 6 '-G L$ & $\operatorname{Gal}(\beta 1-6) \mathrm{Gal}(\beta 1-4) \mathrm{Glc}$ \\
\hline 9 & novo-LNPI & $\left.\begin{array}{l}\operatorname{Gal}(\beta 1-4) \operatorname{GlcNAc}(\beta 1-6) \\
\operatorname{Gal}(\beta 1-3)\end{array}\right) \operatorname{Gal}(\beta 1-4) \mathrm{Glc}$ \\
\hline 10 & $\beta 33^{\prime}-$ GalNAcL & GalNAc( $(\beta 1-3)$ Gal $(\beta 1-4)$ Glc \\
\hline 11 & $\beta 66^{\prime}-$ GlcNAcL & $\operatorname{GlcNAc}(\beta 1-6) \operatorname{Gal}(\beta 1-4) \mathrm{Glc}$ \\
\hline 12 & $\mathrm{LN} n \mathrm{~T}$ & $\operatorname{Gal}(\beta 1-4) \operatorname{GlcNAc}(\beta 1-3) \operatorname{Gal}(\beta 1-4) \mathrm{Glc}$ \\
\hline 13 & $\mathrm{LN} n \mathrm{H}$ & $\begin{array}{l}\operatorname{Gal}(\beta 1-4) \operatorname{GlcNAc}(\beta 1-6) \backslash \\
\operatorname{Gal}(\beta 1-4) \operatorname{GlcNAc}(\beta 1-3)\end{array}$ \\
\hline
\end{tabular}

Acidic oligosaccharides

14 3'-O-Lac phosphate

15 3'S Gal

16 3'-SL

$\mathrm{Gal}(\beta 1-4) \mathrm{Glc}^{\prime} \mathbf{3}^{-}-\mathrm{PO}_{4}$

$17 \quad 6$ '-SL

18 3'-Neu5GcL

$\operatorname{Neu} 5 \mathrm{Ac}(\alpha 2-3) \mathrm{Gal}$

19 6'-Neu5GcL

$\operatorname{Neu} 5 \mathrm{Ac}(\alpha 2-3) \mathrm{Gal}(\beta 1-4)$ Glc

Neu5Ac( $\alpha 2-6)$ Gal( $\beta 1-4) G l c$

Neu5Gc( $\alpha 2-3)$ Gal $(\beta 1-4)$ Glc

Neu5Gc( $\alpha 2-6)$ Gal $(\beta 1-4)$ Glc

20 6'-SLacNAc

Neu5Ac( $\alpha 2-6) \operatorname{Gal}(\beta 1-4)$ GlcNAc

21 6'-Neu5GcLacNAc

Neu5Gc( $\alpha 2-6)$ Gal((31-4)GlcNAc

22

Neu5Ac( $\alpha 2-3) G a l(1-3) G a l(1-4) G l c$

23 DSL

$\operatorname{Neu} 5 \mathrm{Ac}(\alpha 2-8) \operatorname{Neu} 5 \mathrm{Ac}(\alpha 2-3) \mathrm{Gal}(\beta 1-4) \mathrm{Glc}$

24

6'-SLacNAc-1-O-phosphate

$\mathrm{Neu} 5 \mathrm{Ac}(\alpha 2-6) \mathrm{Gal}(\beta 1-4)$ GlcNAc-1-PO 4

25

6'-SLacNAc-6-O-phosphate

Neu5Ac( $\alpha 2-6)$ Gal( $(\beta 1-4)$ GlcNAc-6- $\mathrm{PO}_{4}$

bovine colostrum or mature milk will be utilized by industry as functional foods, animal feeds or biomedical products. For example, several reports have suggested that bovine milk oligosaccharides can be used as anti infection materials.

It is thought that adhesion of Neisseria meningitides, a human-specific pathogen causing meningitis and speticemia, is mediated by type IV pili (Hakkarainen et al., 2005). A mictotiter well pili-binding assay was used 
to investigate the binding of type IV pili isolated from $N$. meningitides to different glycoproteins. Inhibition of pili binding to bovine thyroglobulin and human salivary agglutinin by fractionated human and bovine milk oligosaccharides was demonstrated. The binding of Neisseria pili to bovine thyroglobulin was more effective and was clearly inhibited by neutral or acidic bovine milk oligosaccharides at concentrations of 1 2 g 1 , suggesting that these fractions had the potential ability to inhibit the attachment of this bacterium to the colonic mucosa (Hakkarainen et al., 2005).

Fractions containing milk oligosaccharides, in the form of supernatants that had been separated from colostrum and from transitional, mature and late lactation milk of Spanish brown cows by ethanol precipitation and subsequent centrifugation, were used to investigate the inhibition of hemagglutination by seven enterotoxigenic E. coli strains (K99, FK, F41, F17, B16, B23 and B64). These strains had been isolated from diarrheal calves. The fractions from the transitional and mature milk inhibited hemagglutination by all of these strains, whereas those from colostrum and late lactation milk produced weaker inhibition (Martin et al., 2002). It was suggested that this inhibition was due to $3^{\prime}-\mathrm{SL}, 6^{\prime}-\mathrm{SL}, 6^{\prime}-\mathrm{SLN}$ and DSL. The fractions from transitional and mature milk, in which the ratio of $6^{\prime}$-SL to $3^{\prime}$-SL was higher than in the fractions from colostrum and late lactation milk, had a stronger effect than the others

It can be expected that milk oligosaccharides of other domestic farm animals, such as goats, sheep, and camels, will also be used as biofunctional materials. The milk oligosaccharide content of goat milk is $0.25 \sim 0.39$ $\mathrm{g} \Lambda$; this is higher than that of bovine $(0.03 \sim 0.06 \mathrm{~g} \Lambda)$ or ovine (0.02 0.04 $\mathrm{g} \Lambda)$ milk. In addition, the variety of oligosaccharides in goat milk is greater than that in bovine or ovine milk, as shown by the profiles from HPEAC analysis (Martinez-Ferez et al., 2006; Mehra \& Kelly, 2006). Colostrum from the Japanese Saanen breed contains more $6^{\prime}$-SL than $3^{\prime}$-SL; it also contains $6^{\prime}-N$-glycolylneuraminyllactose $\quad(N e u 5 G c(\alpha 2-6) \mathrm{Gal}(\beta 1-$ 4)Glc), Gal $(\alpha 1-3) \mathrm{Gal}(\beta 1-4) \mathrm{Glc}$, $\mathrm{Gal}(\beta 1-3) \mathrm{Gal}(\beta 1-4) \mathrm{Glc}$,

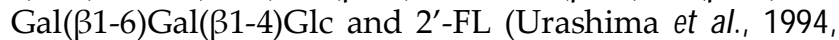
1997). Another study has shown that mature milk from Spanish goats contains 6'-SL, 3'-SL, disialyllactose, Nglycolylneuraminyllactose, 3 '-galactosyllactose, $N$-acetyl glucosaminyllactose, LNH and additional high molecular oligosaccharides, as demonstrated by analysis with FAB-MS, but no fucosyl oligosaccharides (MartinezFerez et al., 2006). Ovine colostrum contains more 3'-N-glycolylneuraminyllactose than 3'-SL and 6'-SL (Nakamura et al., 1998) and, notably, contains Neu5Gc in preference to Neu5Ac.

A recent study on rats showed that goat milk oligosaccharides have an anti-inflammatory effect in the colon (Daddaowa et al., 2006). In this study, colitis was induced by the hapten, trinitrobenzenesulfonic acid (TNBS). The experimental rats (OS) were fed a diet containing 500 $\mathrm{mg} / \mathrm{kg}$ per day of goat milk oligosaccharides, from 2 days prior to the induction until day 6 , after which all the rats were weighed and then killed, the entire colon was removed, opened and scored for visible damage and then divided into several pieces for biochemical determinations. When the OS rats were compared with control rats in which colitis had been induced by TNBS but that had not been treated with oligosaccharides, it was found that the OS rats showed decreased anorexia, reduced loss of body weight, reduced bowel wall thickening and less necrosis of the colon. Biochemically, the OS rats had lower colonic levels of inducible nitric oxide synthase (iNOS), cyclooxygenase 2 (COX2), interleukin$1 \beta$ and mucin 3, as well as increased trefoil factor 3. These results showed that goat milk oligosaccharides are anti-inflammatory when administered as a pretreatment in the TNBS model of rat colitis, most likely due to their action as prebiotics resulting in favorable changes in the colonic bacterial flora. Since TNBS-induced colitis is widely used as a preclinical model of inflammatory bowel disease in humans, it was suggested that goat milk oligosaccharides might be useful in the management of this disease.

\section{CONCLUDING REMARKS}

As the development and improvement of methods for structural determination by using smaller amount of samples as presently required still going on, the new structure of milk oligosaccharides will be still discovered. More studies on the functional biological characteristics of the milk oligosaccharides are still needed. Those study results are needed to confirm milk oligosaccharides role in the various biological processes, as well as to overcome the problems especially in the synthesis of milk oligosaccharides-like compound at the industrial level. The isolation of milk oligosaccharides from the colostrum of domestic animals at industrial scale, for their utilization as biofunctional food and feedstuffs as well as for drugs is still in the early stage. Although still in its infancy, milk oligosaccharides industrial utilization can be expected in the near future.

\section{REFERENCES}

Asakuma, S., M. Akahori, K. Kimura, Y. Watanabe, T. Nakamura, M. Tsunemi, I. Arai, Y. Sanai, \& T. Urashima. 2007. Sialyl oligosaccharides of human colostrum: Changes in concentration during the first three days of lactation. Biosci. Biotech. Biochem. 71: 1447-1451.

Asakuma, S., T. Urashima, M. Akahori, M. Ohbayashi, T. Nakamura, K. Kimura, Y. Watanabe, I. Arai, \& Y. Sanai. 2008. Variation in neutral oligosaccharide levels in human colostrums. Eur. J. Clin. Nutr. 62: 488-494.

Bao, Y., L. Zhu, \& D. S. Newburg. 2007. Simultaneous quantification of sialyloligosaccharides from human milk by capillary electrophoresis. Anal. Biochem. 370: 206-214.

Benno, Y. \& T. Mitsuoka. 1986. The development of gastrointestinal micro-flora in humans and animals. Bifidofact. Microflora 5: 13-25.

Bode, L., S. Rudloff, C. Kunz, S. Strobel, \& N. Klein. 2004 Human milk oligosaccharides reduce platelet-neutrophil complex formation leading to a decrease in neutropil $\beta 2$ integrin expression. J. Leukoc Biol. 76: 820-826.

Bode, L. 2006. Recent advances on structure, metabolism and function of human milk oligosaccharides. J. Nutr. 136: 2127-2130.

Brand-Miller, J.C., P. McVeagh, Y, McNeil, \& M. Messer. 1998. Digestion of human milk oligosaccharides by healthy infants evaluated by the lactulose hydrogen breath test. J. 
Pediatr. 133: 95-98

Chaturvedi, P., C. D. Warren, M. Altaye, A. L. Morrow, G. Ruiz-Palacios, L. K. Pickering, \& D. S. Newburg. 2001. Fucosylated human milk oligosaccharides vary between individuals and over the course of lactation. Glycobiology 11: 365-370.

Coppa, G. V., P. Pierani, L. Zampini, I. Carloni, A. Carlucci, C. Catacci, \& O. Gabrielli. 1999. Oligosaccharides in human milk during different phases of lactation. Acta Paediat. Suppl. 430: 89-94.

Coppa, G. V., L. Zampini, T. Galeazzi, B. Facinelli, L. Ferrante, R. Carpetti, \& G. Orazio. 2006. Human milk oligosaccharides inhibit the adhesion to caco-2 cells of diarrhea pathogens: Escherichia coli, Vibrio cholerae, and Salmonella fyris. Pediatr. Res. 59: 377-382.

Daddaowa, A., V. Puerta, P. Requena, A. Martinez-Ferez, E. Guadix, Sanchez de Mediza, A. Zarzuelo, M. Dolerez Svarez, J. Josa Boya, \& O. Martinez-Augustin. 2006. Goat milk oligosaccharides are anti-inflammatory in rats with hapten-induced colitis. J. Nutr. 136: 672-675.

Derensy-Dron, D., F. Krzewinski, C. Brassart, \& S. Bouquelet. 1999. $\beta-1,3-G a l a c t o s y l-N$-acetylhexosamine phosphorylase from Bifidobacterium bifidum DSM 20082: characterization, partial purification and relation to mucin degradation. Biotechnol. Appl. Biochem. 29: 3-10.

Engfer, M. B., B. Stahl, B. Finke, G. Sawatzki, \& H. Daniel. 2000. Human milk oligosaccharides are resistant to enzymatic hydrolysis in the upper gastrointestinal tract. Am. J. Clin. Nutr. 71: 1589-1596.

Gnoth, M. J., S. Rudloff, C. Kunz, \& R. K. H. Kinne. 2001. Investigations of the in vitro transport of human milk oligosaccharides by Caco-2 monolayer using a novel high performance liquid chromatography-mass spectrometry technique. J. Biol. Chem. 276: 34363-34370.

Gopal, P. K. \& H. S. Gill. 2000. Oligosaccharides and glycoconjugates in bovine milk and colostrums. Br. J. Nutr. 84: S69-S74

Hakkarainen, J., M. Toivanen, A. Leinonen, L. Frangsmyr, N. Stronberg, S. Lapinjoki, X. Nassif, \& C. TikkanenKaukanen. 2005. Human and bovine milk oligosaccharides inhibit Neisseria meningitidis pili attachment in vitro, J. Nutr. 135: 2445-2448.

Hong, P., M. R. Ninonuevo, B. Lee, C. Lebrilla, \& L. Bode. 2009. Human milk oligosaccharides reduce HIV-1-gp120 binding to dendritic cell-specific ICAM3-grabbing nonintegrin (DC-SIGN). Br. J. Nutr. 101: 482-486.

Johansson, P., J. Nilsson, J. Angstrom, \& H. Miller-Pandraza. 2005. Interaction of Helicobacter pylori with sialylated carbohydrates: the dependence on different parts of the binding trisaccharide Neu5Ac $\alpha 3$ Gal $\beta 4$ GlcNAc. Glycobiology 15: 625-636.

Katayama, T., A. Sakuma, T. Kimura, Y. Makimura, J. Hiratake, K. Sakata,, T. Yamanoi, H. Kumagai, \& K. Yamamoto. 2004. Molecular cloning and characterization of Bifidobacterium bifidum 1,2- $\alpha$-L-fucosidase (AfcA), a novel inverting glycosidase (glycoside hydrolase family 95). J. Bacteriol. 186: 4885-4893.

Kitaoka, M., J. Tian, \& M. Nishimoto. 2005. Novel putative galactose operon involving lacto- $N$-biose phosphorylase in Bifidobacterium longum. Appl. Environ. Microbiol. 71: 3158-3162.

Klein, A., A. Schwertman, M. Peters, C. Kunz, \& S. Strobel. 2000. Immunomodulatory effects of breast milk oligosaccharides. In: Short and Long Term Effects of Breast Feeding on Child Health (B. Koletzki, ed.), pp. 251-259, Kluwer Academic Plenum Publishers, New York.

Kunz, C., S. Rudloff, A. Hintelmann, G. Pohlentz, \& H. Egge. 1996. High-pH anion-exchange chromatography with pulsed amperometric detection and molar response fac- tors of human milk oligosaccharides. J. Chromatogr. B 685: 311-221.

Kunz C, G. M. Rodriguez-Palmero, B. Koletzko, \& R. Jensen. 1999. Nutritional and biochemical properties of human milk, Part I: General aspects, proteins, and carbohydrates. Clin Perinatol 26, 307-333.

Leo, F., S. Asakuma, T. Nakamura, K. Fukuda, A. Senda, \& T. Urashima. 2009. Improved determination of milk oligosaccharides using a single derivatization with anthranilic acid and separation by revsersed-phase highperformance liquid chromatography. J. Chromatogr. A. 1216: 1520-1523.

Leo, F., S. Asakuma, K. Fukuda, A. Senda, \& T. Urashima. 2010. Determination of sialyl and neutral oligosaccharide levels in transition and mature milks of Samoan women, using anthranilic derivatization followed by reverse phase high performance liquid chromatography. Biosci. Biotech. Biochem. 74: 298-303.

LoCascio, R. G., M. R. Ninonuevo, S. L. Freeman, D. A. Sela., R. Grimm, C. B. Lebrilla, D. A. Mills, \& J. B. German. 2007. Glycoprofiling of bifidobacterial consumption of human milk oligosaccharides demonstrates strain specific, preferential consumption of small chain glycans secreted in early human lactation. J. Agric. Food. Chem. 55: 8914-8919.

Martin, M. J., S. Martin-Sosa, \& P. Hueso. 2002. Binding of milk oligosaccharides by several enterotoxigenic Escherichia coli strains isolated from calves. Glycoconj. J. 19: 5-11.

Martinez-Ferez, A., S. Rudloff, A. Guadix, C. A. Henkel, G. Pohlentz, J. J. Boza, E. M. Guadix, \& C. Kunz. 2006. Goat's milk as a natural source of lactose-derived oligosaccharides isolation by membrane technology. Int. Dairy J. 16: 173-181.

Mehra, R. \& P. Kelly. 2006. Milk oligosaccharides: Structural and technological aspects. Int. Dairy J. 16: 1334-1340.

Messer, M. \& T. Urashima. 2002. Evolution of milk oligosaccharide and lactose. Trends Glycosci. Glycotech. 14: 153-176.

Nakamura, T., T. Urashima, M. Nakagawa, \& T. Saito. 1998. Sialyllactose occurs as free lactones in ovine colostrum. Biochim. Biophys. Acta 1381: 286-292.

Nakamura, T., H. Kawase, K. Kimura, Y. Watanabe, M. Ohtani, I. Arai, \& T. Urashima. 2003. Concentrations of sialyl oligosaccharides in bovine colostrum and milk during the prepartum and early lactation. J. Dairy Sci. 86: 1315-1320.

Newburg, D. S. \& S. H. Naubauer. 1995. Carbohydrates in milks: Analysis, quantities and significance. In: Handbook of Milk Composition (R, G. Jensen ed.), pp. 273-349, Academic Press, San Diego.

Newburg, D. S. 2000. Oligosaccharides in human milk and bacterial colonization. J. Pediatr. Gastroenterol. Nutr. 30: S8-S17.

Ninonuevo, M. R., Y. Park, H. Yin, J. Zhang, R. E. Ward, B. H. Clowers, J. B. German, S. L. Freeman, K. Killeen, R. Grimm, \& C. B. Lebrilla. 2006. A strategy for annotating the human milk glycome. J. Agric. Food Chem. 54: $7471-7480$.

Nishimoto, M. \& M. Kitaoka. 2007. Identification of $N$ acetylhexosamine 1-kinase in the complete lacto- $N$-biose $\mathrm{I} /$ galacto- $N$-biose metabolic pathway in Bifidobacterium longum. Appl. Environ. Microbiol. 73: 6444-6449.

Obermeier, S., S. Rudloff, S. Pohlentz, M. J. Lentze, \& C. Kunz. 1999. Secretion of ${ }^{13} \mathrm{C}$-labelled oligosaccharides into human milk and infants urine after an oral $\left[{ }^{13} \mathrm{C}\right]$ galactose load. Isotopes Environ. Haelth Stud. 35: 119-125.

Rotimi, V. O. \& B. I. Duerden. 1981. The development of the bacterial flora in normal neonates. J. Med. Microbiol. 14: 
51-58.

Rudloff, S., G. Pohlentz, L. Diekmann, H. Egge, \& C. Kunz. 1996. Urinary excretion of lactose and oligosaccharides in preterm infants fed human milk or infant formula. Acta Paediatr. 85: 598-603.

Ruiz-Palacios, G. M., L. E. Cervantes, P. Ramos, B. ChavezMunguia, \& D. S. Newburg. 2003. Campylobacter jejuni binds intestinal $\mathrm{H}(\mathrm{O})$ antigen (Fuc $\alpha$ 1, 2Gal $\beta$ 1, 4GlcNAc), and fucosyloligosaccharides of human milk inhibit its binding and infection, J. Biol. Chem. 278: 14112-14120.

Schumacher, G., G. Bendas, B. Stahl, \& C. Beermann. 2006. Human milk oligosaccharides affect P-selectin binding capacities: In vitro investigation. Nutr. 22: 620-627.

Sela, D. A., J. Chapman, A. Adeuya, J. H. Kim, F. Chen, T. R. Whitehead, A. Lapidus, D. S. Rokhsar, C. B. Lebrilla, \& J. B. German. 2008. The genome sequence of Bifidobacterium longum subsp. infantis reveals adaptations for milk utilization within the infant microbiome. Proc. Natl. Acad. Sci. U S A 105: 18964-18969.

Shen, Z., C. D. Warren, \& D. S. Newburg. 2000. High-performance capillary electrophoresis of sialylated oligosaccharides of human milk. Anal. Biochem. 279:37-45.

Suzuki, R., J. Wada, T. Katayama, S. Fushinobu, T. Wakagi, H. Shoun, H. Sugimoto, A. Tanaka, H. Kumagai, H. Ashida, M. Kitaoka, \& K. Yamamoto. 2008. Structural and thermodynamic analyses of solute-binding Protein from Bifidobacterium longum specific for core 1 disaccharide and lacto-N-biose I. J. Biol. Chem. 283: 13165-13173.

Tao, N., E. J. DePeters, S. Freeman, J. B. German, R. Grimm, \& C. B. Lebrilla. 2008. Bovine milk glycome. J. Dairy Sci. 91: 3768-3778.

Thurl, S., B. Muller-Wermer, \& G. Sawatzki. 1996. Quantification of individual oligosaccharide compounds from human milk using high-pH anion exchang chromatography. Anal. Biochem. 235: 202-206.

Urashima, T., W. A. Bubb, M. Messer, Y. Tsuji, \& Y. Taneda. 1994. Studies of the neutral trisaccharides of goat (Capra hircus) colostrum and of the one- and two-dimensional ${ }^{1} \mathrm{H}$ and ${ }^{13} \mathrm{C}$ NMR spectra of 6 '- $\mathrm{N}$-acetylglucosaminyllactose. Carbohydr. Res. 262: 173-184.

Urashima, T., S. Murata, \& T. Nakamura. 1997. Structural determination of monosialyl trisaccharide obtained from caprine colostrum. Comp. Biochem. Physiol. 116B: 431-435.

Urashima, T., T. Saito, T. Nakamura, \& M. Messer. 2001. Oligosaccharides of milk and colostrum in non-human mammals. Glycoconj. J. 18: 357-371.

Urashima, T., S. Asakuma, \& M. Messer. 2007. Milk oligosaccharides. In: J.P. Kamerling, G.-J. Boons, Y.C. Lee, A. Suzuki, N. Taniguchi \& A.G.J. Voragen (eds.). Comprehensive Glycoscience. Elsevier, Amsterdam. Vol. 4, pp. 695-724.

Urashima, T., G. Odaka, S. Asakuma, Y. Uemura, K. Goto, A. Senda, T. Saito, K. Fukuda, M. Messer, \& O. T. Oftedal. 2009. Chemical characterization of oligosaccharides in chimpanzee, bonobo, gorilla, orangutan, and siamang milk or colostrum. Glycobiology 19: 499-508 doi:10.1093/glycob/cwp006.

Urashima, T., M. Kitaoka, T. Terabayashi, K. Fukuda, M. Ohnishi, \& A. Kobata. (in press). Milk oligosaccharides. In: N.S. Gordon (ed.). Oligosaccharides: Sources, Properties and Applications. Nova Science Publishers, Inc. New York. pp. 1-77.

Veh, R. W., J. C. Michalski, A. P. Corfield, M. Sander-Wewer, D. Gies, \& R. Schauer. 1981. New chromatographic system for the rapid analysis and preparation of colostrums sialyloligosaccharides. J Chromatogr 212: 313-322.

Ward, R. E., M. Ninonuevo, D. A. Mills, C. B. Lebrilla, \& J. B. German. 2007. In vitro fermentability of human milk oligosaccharides by several strains of bifidobacteria. Mol. Nutr. Food Res. 51: 1398-1405.

Wada, J., T. Ando, M. Kiyohara, H. Ashida, M. Kitaoka, M. Yamaguchi, H. Kumagai, T. Katayama, \& K. Yamamoto. 2008. Bifidobacterium bifidum lacto- $N$-biosidase, a critical enzyme for the degradation of human milk oligosaccharides with a type 1 structure. Appl. Environ. Microbiol. 74: 3996-4004. 